\title{
A combined small- and wide-angle x-ray scattering detector for measurements on reactive systems
}

Linda Vallenhag', Sophie E. Canton, Peter Sondhauss, Dörthe Haase, and Frederik Ossler

Citation: Review of Scientific Instruments 82, 083104 (2011); doi: 10.1063/1.3613958

View online: http://dx.doi.org/10.1063/1.3613958

View Table of Contents: http://aip.scitation.org/toc/rsi/82/8

Published by the American Institute of Physics

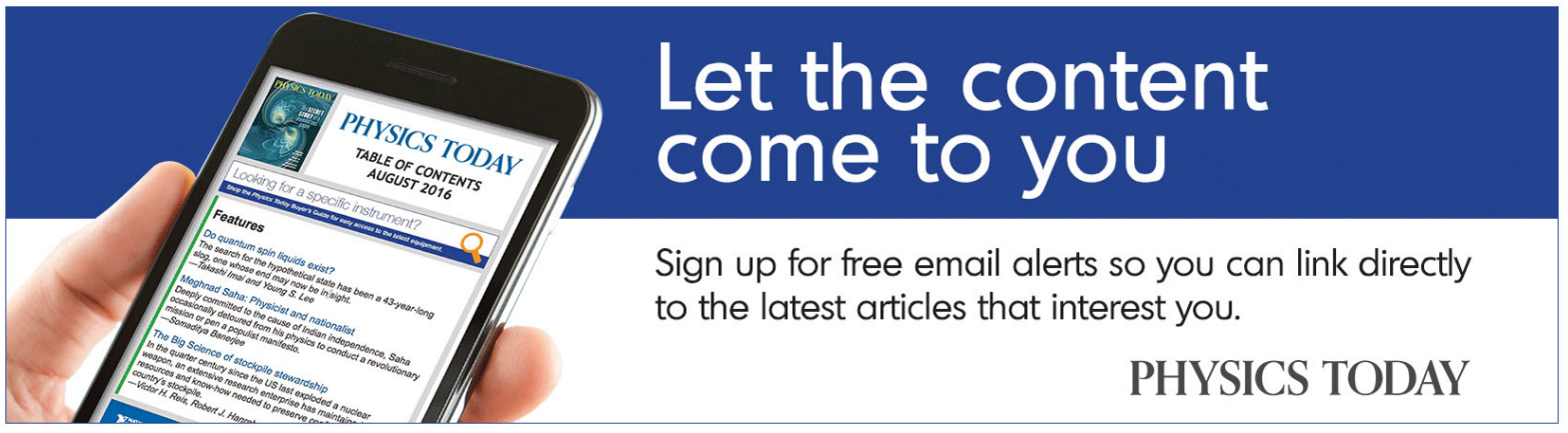




\title{
A combined small- and wide-angle x-ray scattering detector for measurements on reactive systems
}

\author{
Linda Vallenhag, ${ }^{1, \text { a) }}$ Sophie E. Canton, ${ }^{2}$ Peter Sondhauss, ${ }^{2}$ Dörthe Haase,$^{2}$ \\ and Frederik Ossler ${ }^{1}$ \\ ${ }^{1}$ Division of Combustion Physics, Lund University, SE-221 00 Lund, Sweden \\ ${ }^{2}$ MAX-lab, Lund University, SE-221 00 Lund, Sweden
}

(Received 14 April 2011; accepted 29 June 2011; published online 10 August 2011)

\begin{abstract}
A detector with high dynamic range designed for combined small- and wide-angle $\mathrm{x}$-ray scattering experiments has been developed. It allows measurements on single events and reactive systems, such as particle formation in flames and evaporation of levitating drops. The detector consists of 26 channels covering a region from $0.5^{\circ}$ to $60^{\circ}$ and it provides continuous monitoring of the sampled signal without readout dead time. The time resolution for fast single events is about $40 \mu \mathrm{s}$ and for substances undergoing slower dynamics, the time resolution is set to 0.1 or $1 \mathrm{~s}$ with hours of continuous sampling. The detector has been used to measure soot particle formation in a flame, burning magnesium and evaporation of a toluene drop in a levitator. The results show that the detector can be used for many different applications with good outcomes and large potential. () 2011 American Institute of Physics. [doi:10.1063/1.3613958]
\end{abstract}

\section{INTRODUCTION}

Design and manufacturing of nanoparticles and nanostructured materials with special physicochemical properties and functionalities are crucial for the development of electronics, energy conversion, and bioapplications. ${ }^{1-7}$ Particle synthesis can be done in combustion processes, e.g., silica, titania, and iron oxide nanoparticles have been produced in flames using hydrocarbon fuels. ${ }^{8-11}$ One of the most promising tools for particle dynamics studies is based on synchrotron x-ray scattering, ${ }^{12-14}$ which has demonstrated the ability to study soot formation in flames in situ: small-angle x-ray scattering (SAXS) to characterize the size distribution of soot particles, ${ }^{15-21}$ and wide-angle $\mathrm{x}$-ray scattering (WAXS) to study the subnanometer structure. ${ }^{22,23}$ These scattering techniques measure the intensity of scattered photons as a function of the exchanged momentum, $q$, according to

$$
q=\frac{4 \pi}{\lambda} \sin (\theta / 2)
$$

where $\lambda$ is the wavelength of the incident photon and $\theta$ is the scattering angle.

CCD detectors are widely used to record small- and wide-angle x-ray scattering in lab-based or synchrotron-based experiments. ${ }^{24-26}$ A CCD detector with a time resolution down to a millisecond (or milliseconds) has been used to perform SAXS and WAXS experiments. ${ }^{27}$ The PILATUS detector is a two-dimensional detector which is a hybrid pixel detector operating in single-photon-counting mode with a readout time of less than $3 \mathrm{~ms}$ and a frame rate of $200 \mathrm{~Hz}{ }^{28,29}$ The one-dimensional microstrip detector, MYTHEN, developed for time-resolved x-ray powder diffraction experiments has a readout time in the 100-250 $\mu$ s range (4-24 bits), a maximum frame rate of $10-90 \mathrm{~Hz}$ (24-4 bits), and covers $120^{\circ} .^{30}$ A high-speed silicon annular strip detector has been designed

\footnotetext{
a)Electronic mail: Linda.Vallenhag@ forbrf.lth.se.
}

and built for time-resolved small-angle $\mathrm{x}$-ray scattering experiments at the Advanced Photon Source of Argonne National Laboratory. ${ }^{31,32}$ The detector consists of 128 concentric rings operating as individual detector elements. The output signals are then given as a function of the corresponding q-values of the rings, providing one-dimensional scattering information. The frame rate is $271 \mathrm{kHz}$ and within this time, a maximum of 64 analog-to digital samples per channel are averaged and stored. A drawback for these systems is the considerable readout time.

In this work we present a combined multi-slit-channel (MSC) SAXS/WAXS detector, which has a high dynamic range and almost no readout dead time. The detector includes a 20-channel SAXS detector and a 6-channel WAXS detector, particularly adapted for combustion and flame dynamics studies enabling continuous measurements for hours sampling every $5 \mu$ s and signal processing on-line typically every 0.1 or $1 \mathrm{~s}$ before storage in the computer memory. We report experimental results on flames and reactive systems, e.g., magnesium combustion. Besides the parallel acquisition system, advantages are the compactness requiring no more than approximately $50 \mathrm{~cm} \times 50 \mathrm{~cm} \times 50 \mathrm{~cm}$ of lab space and the strong discrimination against background scattering by spatial filtering. The system is meant to be transportable to different synchrotron beamlines throughout the world.

\section{DETECTOR LAYOUT}

The MSC-SAXS and WAXS detectors consist of, respectively, 20 and 6 channels for simultaneous sampling over a range from $0.5^{\circ}$ to $10^{\circ}$ and $17.5^{\circ}$ to $60^{\circ}$. The angular steps are $0.5^{\circ}$ and $8.5^{\circ}$, respectively. Every channel is defined by two slits. A $3 \mu \mathrm{m}$ thick $\mathrm{Al}$ foil covers the front slits and it is used to suppress background from visible light. The scintillators (ZnS:Ag) convert the entering x-ray radiation into visible radiation in the blue wavelength region. The distances 
from the front slits to the focus are $150 \mathrm{~mm}$ for the MSCSAXS detector and $50 \mathrm{~mm}$ for the MSC-WAXS detectors. Every slit on the back of the wide-angle detector leads directly to a photomultiplier tube (Hamamatsu H6780) while the slits of the small-angle detector lead to optical fibers with a length of $2 \mathrm{~m}$ and a diameter of $1 \mathrm{~mm}$ that guide the luminescence from the scintillator to a photomultiplier tube (PMT), which register the signal. A trans-impedance amplifier reads the PMTs outputs. The signals are sampled by two $\mathrm{A} / \mathrm{D}$ acquisition cards that allow a maximum sampling rate of $204.8 \mathrm{k} \mathrm{sample} \mathrm{s}^{-1}$ corresponding to a time resolution close to $5 \mu \mathrm{s}$. The cards have 16 analog input channels each and a 24-bit resolution over a -10 to $+10 \mathrm{~V}$ range, giving a measurement accuracy of $1.19 \mu \mathrm{V}$.

Figure 1 shows a schematic picture of the intersection between the X-ray beam and the volume which is seen by one of the channels. Every slit has a specified width: $\mathrm{w}_{\text {in }}$ is the width of the entrance slit and is smaller than the width of the exit slit, $\mathrm{w}_{\text {out }}$. The height of the slits $h$ is the same for both the entrance and exit slits. $\alpha$ is the broadening of the sampling angle $\theta$ due to the slit width and this results in an extension in z-direction. This so-called depth of view is largest for the smallest sampling angles and in combination with the height, $\mathrm{h}_{\text {beam }}$, and the width, $\mathrm{w}_{\text {beam }}$, of the $\mathrm{x}$-ray beam it determines the measured volume $V_{i}$ (the darker area of the x-ray beam in Fig. 1) for every channel $i$. This volume is largest for the smallest angles and the beam size has to be small in order to keep it minimized. In a perfect situation the angular broadening and the beam size would be very small, resulting in a point-like measured volume for every channel, as can be seen in Fig. 1. When measuring on objects that are larger than the depth of view, the smallest channel measures a wider volume, extending outside the range of the object to be investigated. The contribution from air outside the range of the object needs to be subtracted in order to obtain the scattering

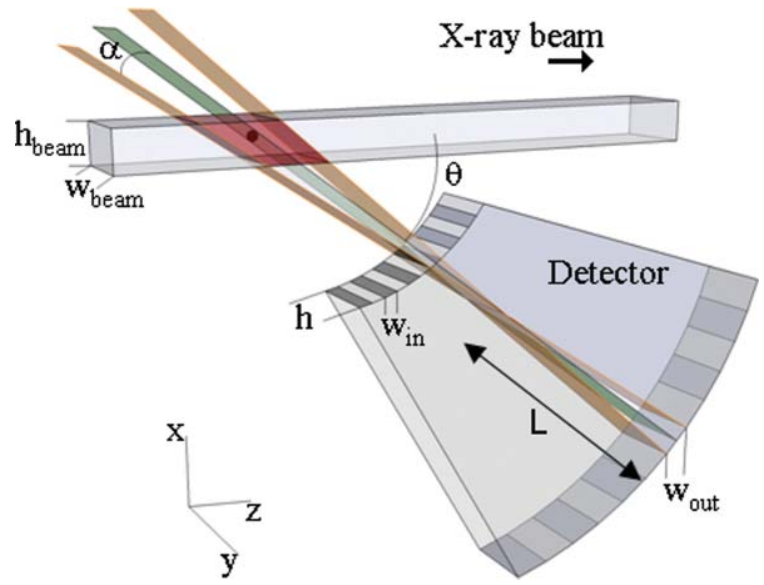

FIG. 1. (Color online) The intersection between the x-ray beam and the visible volume for one of the channels of the detector. The height and width of the $\mathrm{x}$-ray beam are $\mathrm{h}_{\text {beam }}$ and $\mathrm{w}_{\text {beam }}$, respectively. The height of the slit is $\mathrm{h}$. $\mathrm{w}_{\text {in }}$ and $\mathrm{w}_{\text {out }}$ are the entrance and the exit widths of the slits respectively. $\theta$ is the sampling angle for the channel shown and $\alpha$ is half of the acceptance angle of the sampling channel determined by the widths of the slits. $\mathrm{L}$ is the distance from the exit slit to the intersection of the extreme angle rays inside the detector. The intersection region is the scattering volume for the channel shown.

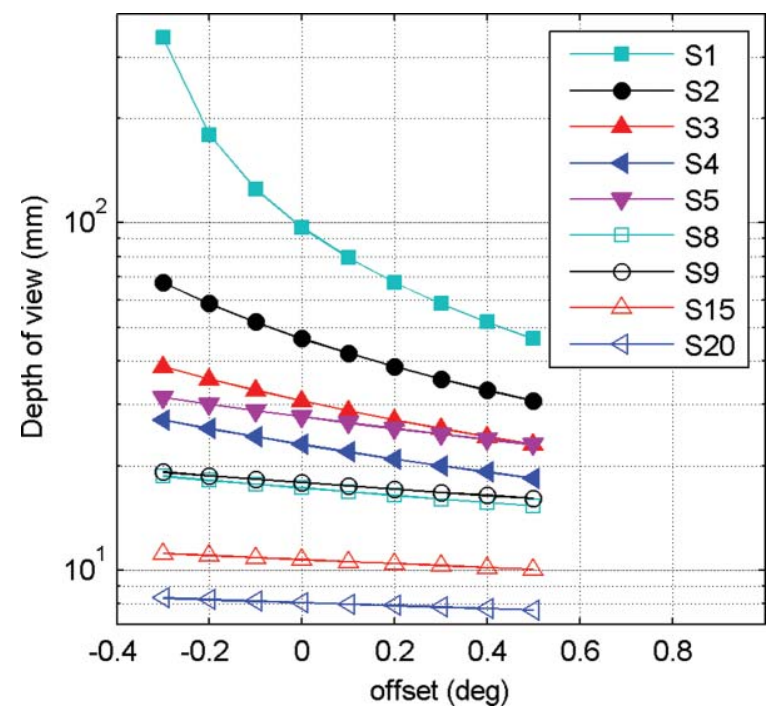

FIG. 2. (Color online) The depth of view as a function of the tilt angle for some channels of the MSC-SAXS detector.

intensity $I_{\text {object, }, i}$ from the object at channel $i$

$$
\begin{aligned}
I_{\text {object }, i}= & \left(I_{i}-I_{\text {air }, i} \times\left(V_{i}-V_{\text {object }, i}\right) / V_{i}\right) \\
& \times \frac{V_{i}}{V_{\text {object }, i}} \quad i=1, \ldots, n
\end{aligned}
$$

where $n$ is the number of channels, $I_{\text {object }, i}$ is the scattering per unit volume from the object, $I_{i}$ is the total scattering, $I_{\text {air }, i}$ is the scattering from air, and $V_{i}$ (resp. $V_{\text {object }, i}$ ) are the total scattering volume (resp. the volume of the object).

The depth of view is largely dependent on the scattering angle and independent of the beam size. This means that an angular offset, e.g., for a slightly rotated detector position, would affect the first channels of the MSC-SAXS detector the most. The depth of view has been calculated for the MSCSAXS detector for different angular offsets and the results for 9 different channels are shown in Fig. 2. In order to obtain sufficient spatial resolution while maintaining high enough signal levels the width of the entrance slit for the MSC-SAXS detector increases with scattering angle: (Ch.1-4) $0.2 \mathrm{~mm}$; (Ch.5-8) $0.3 \mathrm{~mm}$; and (Ch.9-20) $0.4 \mathrm{~mm}$. This width is $2 \mathrm{~mm}$ for all the channels of the MSC-WAXS detector.

\section{A. Operational modes of the detector}

The detection system can work in three different modes depending on the layout of the experiment. The first operation mode (I) is when both the angle and the wavelength are fixed. This gives the highest time resolution and is suitable for studies requiring high dynamic range.

In the second operation mode (II) the angle is kept fixed while the wavelength is changed. This is performed for substances in a steady state or undergoing slow dynamics. This mode is also used for calibration of the angular position of the slits, as described later on.

Studies on substances in steady states can also be conducted with the third operation mode (III), where the wavelength is held fixed while the angle is changed. This mode has 
been used in Refs. 22 and 23 where the MSC-WAXS detector was rotated.

\section{EXPERIMENTAL SET-UP FOR X-RAY SCATTERING}

A schematic layout of the experimental setup used for the flame experiment is shown in Fig. 3. A scintillator combined with a PMT is used to measure the intensity of the incident $\mathrm{x}$-ray radiation. To allow for real-time background subtraction, the signal is periodically by a chopper. A pinhole is placed in the beam after the chopper in order to narrow the beam size. The measurements take place in the focus of the detector, which is the intersection of all the slits. The burner was a small non-cooled porous bronze plug with a diameter of $2 \mathrm{~cm}$ fed with ethylene burning as a yellow diffusion flame. A metal plate was placed above the burner to stabilize the flame and to grow soot on it. The x-ray beam passed through the flame close to $1 \mathrm{~mm}$ below the plate.

The experiments were performed at beamlines D611 and I911-5 at MAX-lab, Lund, Sweden. The longitudinal coherence length of beamline D611 was $10 \mathrm{~nm}$ and the transverse coherence length was approximately $40 \mu \mathrm{m}$. The photon flux was around $10^{12}$ photon $\mathrm{s}^{-1}$. The longitudinal and the transverse coherence lengths for the beamline I911-5 were $90 \mathrm{~nm}$ and $1 \mu \mathrm{m}$, respectively. The photon flux was approximately $10^{10}$ photon $\mathrm{s}^{-1}$.

\section{SIGNAL PROCESSING}

The data acquisition and the processing of the sampled signals were computer controlled with LabVIEW. The computer (NI PXI-1042) had a dual core processor enabling programs to be executed in parallel, meaning that data acquisition and processing could be run at the same time on each processor and that data were sampled continuously without processing dead time.

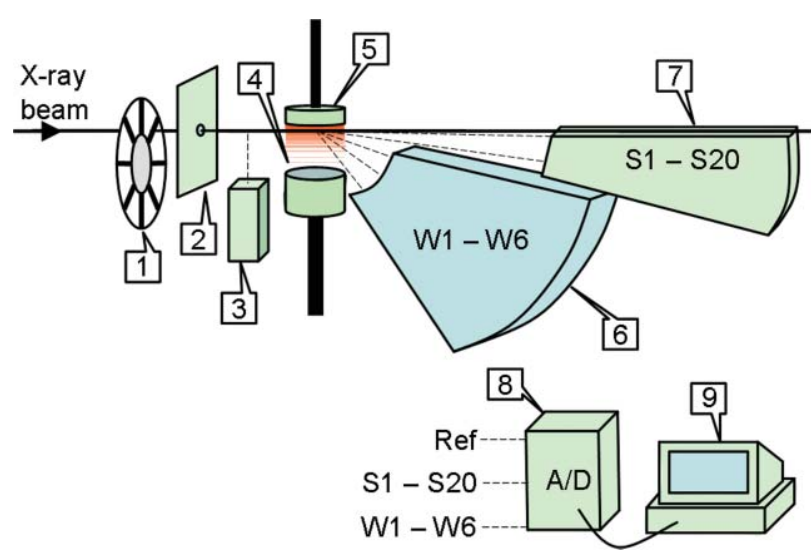

FIG. 3. (Color online) The experimental set-up for flame measurements (1) Chopper, (2) pinhole, (3) reference PMT, (4) burner and flame, (5) metal plate, (6) MSC-WAXS detector, (7) MSC-SAXS detector, (8) transimpedance amplifier, and (9) computer with A/D acquisition cards.
The scattered intensity $I_{i}$ at channel $i$ can be described by the following equations, based on previous works: ${ }^{23,33}$

$$
\begin{aligned}
I_{i}(t, q(\lambda, \theta), \theta, \varphi)= & I_{0} c_{i} r_{e}^{2} p(\theta, \varphi) N(t) \sum_{j=1}^{M} x_{j}(t) \\
& \times\left[\frac{d \sigma_{s j}\left(q_{i}\right)}{d \Omega}\right] \Delta \Omega_{i} \Delta V_{i} \\
& i=1, \ldots, n
\end{aligned}
$$

where $I_{0}$ is the irradiance of the source, $n$ is the number of channels, $t$ is the time of the measurement, $\theta$ is the scattering angle, and $\varphi$ is the angle between the scattering plane and the plane of polarization, which is $90^{\circ}$ in these synchrotron-based experiments. $c_{i}$ is the calibration factor for channel $i, r_{e}$ is the electron radius, $N$ is the total concentration of species, $x_{j}$ is the mole fraction of species $j$, and $d \sigma_{s j}\left(q_{i}\right) / d \Omega$ is its differential scattering cross section. For $\varphi=90^{\circ}$ is $p(\theta, \varphi)=1, \Delta V_{i}$ is the measured volume of channel $i$ and $\Delta \Omega_{i}$ is the solid angle of channel $i$. The solid angle is approximated as follows:

$$
\Delta \Omega \approx \frac{w_{\text {out }} \times h}{L^{2}}
$$

where the parameters are shown in Fig. 1.

The time resolution due to the sampling rate of the data acquisition cards is $4.88 \mu \mathrm{s}$. However, the time resolution also depends on the decay time of the afterglow of the scintillator and the time constant for the amplifier. Part of raw data from the MSC-SAXS detector before the signal processing can be seen in Fig. 4, where the peaks are from photons recorded by the PMTs. There is no background light entering the detector. The inset in the figure shows an enlargement of two photon peaks. The rise and fall times are defined as the time between changes from $10 \%$ to $90 \%$ and changes from $90 \%$ to $10 \%$ of the peak height and they are around $8 \mu \mathrm{s}$ and $45 \mu \mathrm{s}$, respectively. The peak tails have an exponential decay time of $35 \mu \mathrm{s}$.

Two different signal processing methods have been applied, referred to as averaging (AV) and on line background subtraction (OLBS) averaging. The OLBS method is a software designed to operate as lock-in amplifier that reduces the contribution from background radiation by using a periodic signal input and a chopper reference signal which is a square wave and is used to calculate the frequency of the modulated signal, typically $2 \mathrm{kHz}$. The phase shift between

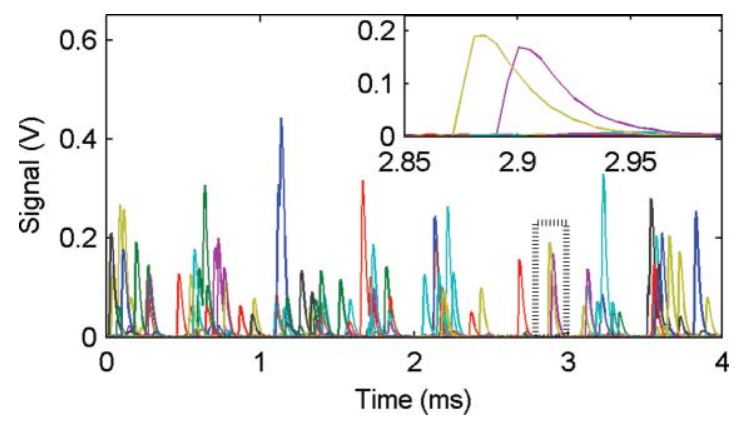

FIG. 4. (Color online) The raw data before signal processing sampled with a sampling rate of $204.8 \mathrm{k} \mathrm{sample} \mathrm{s}^{-1}$. 

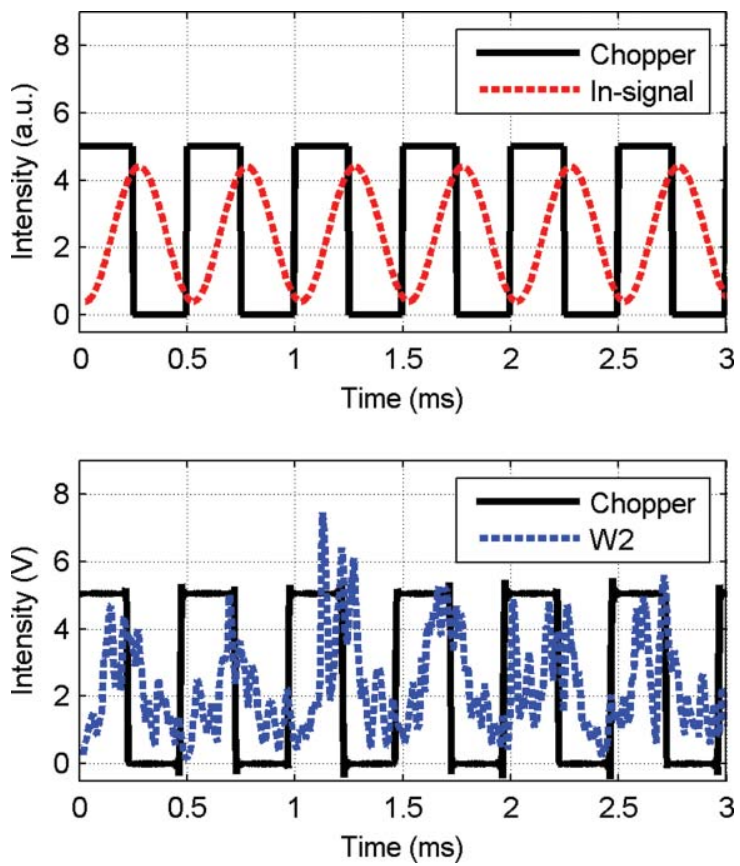

FIG. 5. (Color online) Raw data to be processed by the OLBS method. (a) Simulated data with a phase shift of $110^{\circ}$ between the square wave and the sine wave. (b) Experimental data from the chopper and the two first channels on the MSC-WAXS detector. The frequency of the chopper is $2 \mathrm{kHz}$.

the reference and the modulated signal is found by maximizing the output signal. Figure 5(a) shows a schematic picture of a reference square wave and a sine shaped signal input with a phase shift of $110^{\circ}$. This can be compared to Fig. 5(b) which shows experimental data from the chopper and the second channel from the MSC-WAXS detector where visible background light has entered the detector.

The algorithm calculates the intensity of the incoming signal with subtracted background, $I_{O L B S, i}$, for channel $i$ according to the following formula: ${ }^{34}$

$$
I_{O L B S, i}=\frac{1}{m} \sum_{k=1}^{m}\left(\bar{I}_{2 k}-\frac{1}{2}\left(\bar{I}_{2 k-1}+\bar{I}_{2 k+1}\right)\right) \quad i=1, \ldots, n
$$

where $I_{2 k}$ is the intensity for a peak, $I_{2 k-1}$ and $I_{2 k+1}$ are the intensities of the two dips next to the peak, $m$ is the number of peaks in the signal, and $n$ is the number of channels.

To improve the signal-to-noise level of the measurement that is mainly determined by the photon statistics, the signal is sampled for a specified time determined by the photon flux into the detector. Typically the integration and processing times were $0.1 \mathrm{~s}$ or $1 \mathrm{~s}$ at the highest sampling rate. The OLBS subtracts the background directly while the $\mathrm{x}$-ray beam has to be turned on and off periodically for the AV method in order to subtract the background afterwards. The process time for the $\mathrm{AV}$ is $0.1 \mathrm{~s}$ when sampling with the highest sampling rate for $1 \mathrm{~s}$. This is around 9 times faster than the OLBS, which has a processing time of $0.9 \mathrm{~s}$ for the same condition and with a chopper frequency set to $2 \mathrm{kHz}$.

To compensate for the decrease and fluctuations of the $\mathrm{x}$-ray beam during the run, the raw signals from the scattering data were normalized against the reference signal for the $\mathrm{x}$-ray beam intensity.
Scaling factors for every channel were obtained by comparing the measured scattering for air with theoretical calculations and thereby allow the calibration of the detector.

A more direct way to observe changes in the scattering, without requiring the use of scaling factors, is to plot the ratio $\gamma$, calculated from the data according to the following equation:

$$
\gamma_{i}\left(q_{i}, t\right)=\frac{I_{i}\left(q_{i}, t\right)}{I_{i}\left(q_{i}, t_{0}\right)} \quad i=1, \ldots, n
$$

where $t_{0}$ is the starting time and $n$ is the number of channels. This way of representing the data is used when studying dynamics objects, e.g., flames, in order to easily detect modifications in the system relative to the starting conditions. The quantity $\gamma$ is preferably used whenever the changes relative the initial conditions are significant.

\section{CHARACTERIZATION OF THE DETECTORS}

To calibrate the MSC-SAXS detector, measurements on silver behenate were performed at beamline D611, MAX-lab, Lund, Sweden. The wavelength was scanned in order to get more q-values and find the different peak positions of the sample. The result from a scan between $5 \mathrm{keV}$ and $9 \mathrm{keV}$ is compared with measurements taken with a 2D CCD detector at the Advanced Photon Source at the Argonne National Laboratory, in Fig. 6. An angular offset of $0.3^{\circ}$ was used for the MSC-SAXS detector giving a range from $0.8^{\circ}$ to $10.3^{\circ}$ and the smallest and largest q-values for every channel in the range from $5 \mathrm{keV}$ to $9 \mathrm{keV}$ can be seen in Table I. The MSC-WAXS detector was calibrated with magnesium oxide by scanning the wavelength from 5 to $8.5 \mathrm{keV}$. The angular offset, with respect to the range from $17.5^{\circ}$ to $60^{\circ}$, was determined to be $0^{\circ}$ by comparing with the peaks from magnesium oxide. ${ }^{35}$

Experiments for characterizing the depth of view for the different channels of the MSC-SAXS detector were performed at beamline 1911-5, MAX-lab, Lund, Sweden. The detector was mounted on a translation stage allowing it to be moved in z-direction in Fig. 1. The scattering intensity was measured for different distances from the focus point as a function of the scattering angle. The depth of view for the

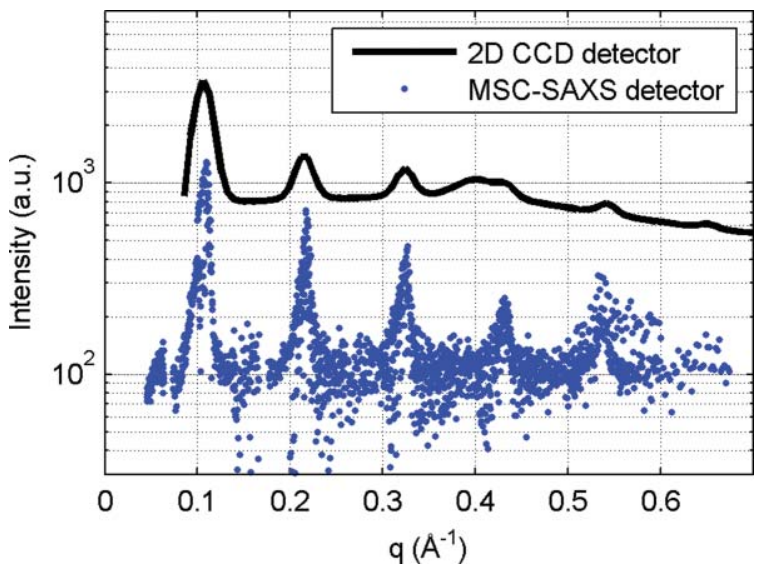

FIG. 6. (Color online) Measurements of silver behenate with the MSCSAXS detector and a 2D CCD detector. 
TABLE I. The smallest and largest q values for every channel in the photon energy range $5 \mathrm{keV}$ to $9 \mathrm{keV}$. The angular offset is $0.3^{\circ}$ for the MSC-SAXS detector.

\begin{tabular}{lccc}
\hline \hline Detector Ch. & Angle $\left(^{\circ}\right)$ & $\mathrm{q}\left(\AA^{-1}\right) @ 5 \mathrm{keV}$ & $\mathrm{q}\left(\AA^{-1}\right) @ 9 \mathrm{keV}$ \\
\hline S1 & 0.8 & 0.035 & 0.064 \\
S2 & 1.3 & 0.057 & 0.10 \\
S3 & 1.8 & 0.080 & 0.14 \\
S4 & 2.3 & 0.10 & 0.18 \\
S5 & 2.8 & 0.12 & 0.22 \\
S6 & 3.3 & 0.15 & 0.26 \\
S7 & 3.8 & 0.17 & 0.30 \\
S8 & 4.3 & 0.19 & 0.34 \\
S9 & 4.8 & 0.21 & 0.38 \\
S10 & 5.3 & 0.23 & 0.42 \\
S11 & 5.8 & 0.26 & 0.46 \\
S12 & 6.3 & 0.28 & 0.50 \\
S13 & 6.8 & 0.30 & 0.54 \\
S14 & 7.3 & 0.32 & 0.58 \\
S15 & 7.8 & 0.34 & 0.62 \\
S16 & 8.3 & 0.39 & 0.66 \\
S17 & 8.8 & 0.39 & 0.70 \\
S18 & 9.3 & 0.41 & 0.74 \\
S19 & 9.8 & 0.43 & 0.78 \\
S20 & 10.3 & 0.45 & 0.82 \\
W1 & 17.5 & 0.77 & 1.39 \\
W2 & 26 & 1.14 & 2.05 \\
W3 & 34.5 & 1.50 & 2.71 \\
W4 & 43 & 1.86 & 3.34 \\
W5 & 51.5 & 2.20 & 3.96 \\
W6 & 60 & 2.53 & 4.56 \\
\hline \hline
\end{tabular}

MSC-SAXS detector was measured as the distance between two points where the intensity of the signal had decreased by $50 \%$ compared to the signal intensity in the focus. The angular offset was $-0.2^{\circ}$ and theoretical calculations of the depth of view using that offset were done and compared with the experimental results, shown in Fig. 7. The jumps observed at channels 5 and 9 are explained by the increase of the slit widths (compare with Fig. 2). This agrees with the measured results, an increase in the depth of view can be seen at channel number 5 and 9 . The ratio between the calculated and the experimental depth of view is 1 for the first channel and almost 2 for the rest of the channels. The first channel is very sensitive to the offset of the detector as can be seen in Fig. 2 displaying the depth of view calculated for different offsets.

The detector has been used for photon energies from $5 \mathrm{keV}$ to $13.6 \mathrm{keV}$.

\section{EXPERIMENTS}

Operation mode I was used in a flame experiment described earlier in Chapter III in relation to Fig. 3. Schematic picture of the dynamics of particle condensing onto the plate is shown in Fig. 8: (a) in the beginning of the experiment the scattering was from gas phase nanoparticles and soot in the flame; (b) gradually particles started to condense on the plate; and (c) the condensed particle layer had grown sufficiently thick (a couple of millimeters) to give a major contribution to the scattering.

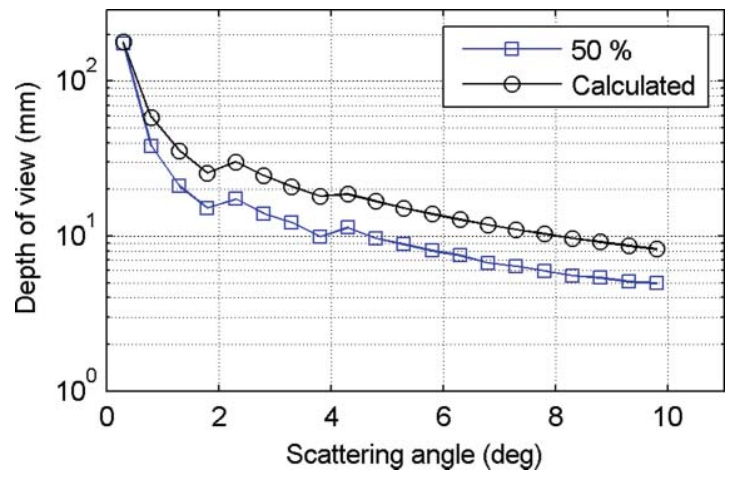

FIG. 7. (Color online) Measured depth of view for the MSC-SAXS detector compared with theoretical calculations.

Figure 9(a) shows the processed signal as a function of time, where data were sampled with the maximum sampling rate for $1 \mathrm{~s}$ using the OLBS method. This is referred to as the time trace. In Fig. 9(a) the signals from all channels are presented, where $S$ refers to the MSC-SAXS detector, $W$ to the MSC-WAXS detector, and Ref. to the reference signal. The photon energy was $5 \mathrm{keV}$ and the detectors were kept fixed.

The signals were averaged over $61 \mathrm{~s}$ at 4 different time positions, marked as A-D in Fig. 9(a), and then plotted in Figs. 9(b) and 9(c) as calibrated intensities and ratio of intensities, respectively, as a function of q. The corresponding q-values can be found in Table I. The ratio $\gamma$ was used in order to visualize changes relative to the initial flame conditions and scattering properties. The 20 data points with smallest qvalues were from the MSC-SAXS detector and the last 6 from the MSC-WAXS detector. The angular offsets were $0.3^{\circ}$ and $0^{\circ}$ for the MSC-SAXS and MSC-WAXS detectors, respectively.

During the first $1000 \mathrm{~s}(\mathrm{~A}-\mathrm{C})$ the temperature increased speeding up the chemistry and the nanoparticles production. The intensity in the SAXS region increased strongly, while maintaining the main shape of the scattering intensity, indicating an increase in particle concentration. Between $1000 \mathrm{~s}$ and $2400 \mathrm{~s}$ (C-D) a peak was observed in the WAXS region

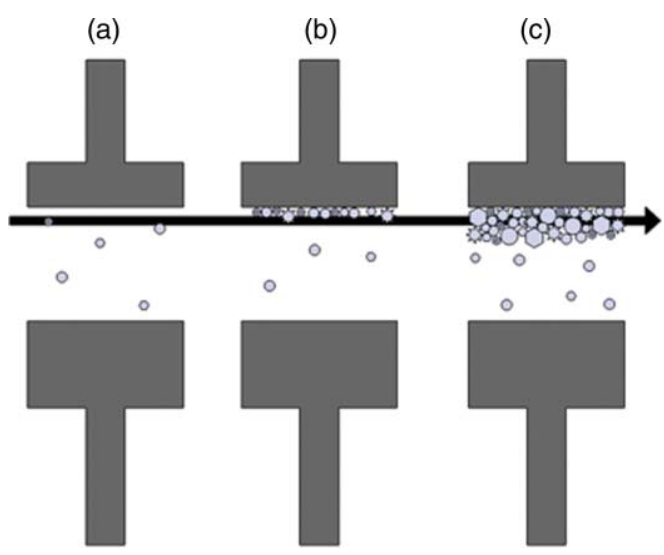

FIG. 8. The x-ray beam is passing through the flame approximately $1 \mathrm{~mm}$ below the plate. (a) Pure monitoring of the gas phase, there is no soot on the plate. (b) Particle starts to deposit on the plate. (c) Monitoring of the condensed phase, which is a couple of millimeters thick. 


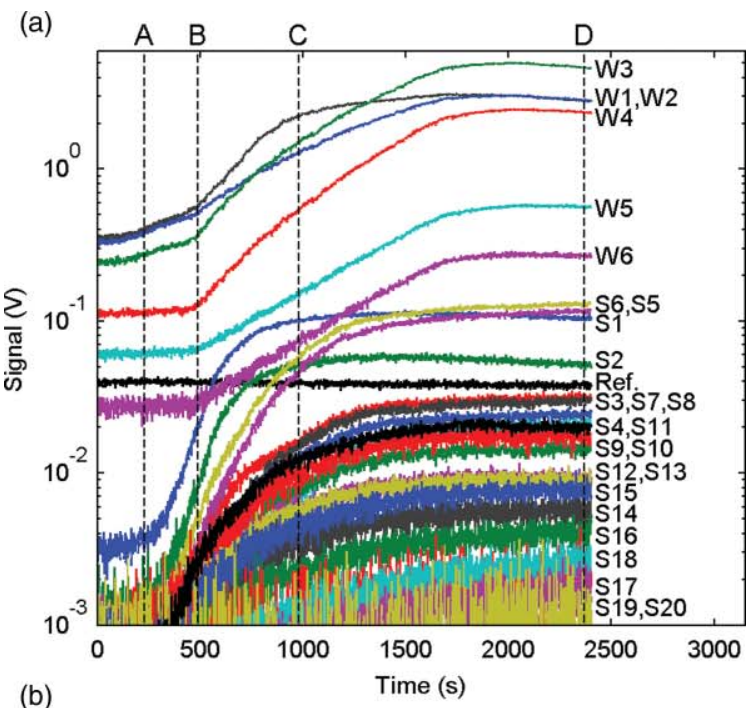

(c)

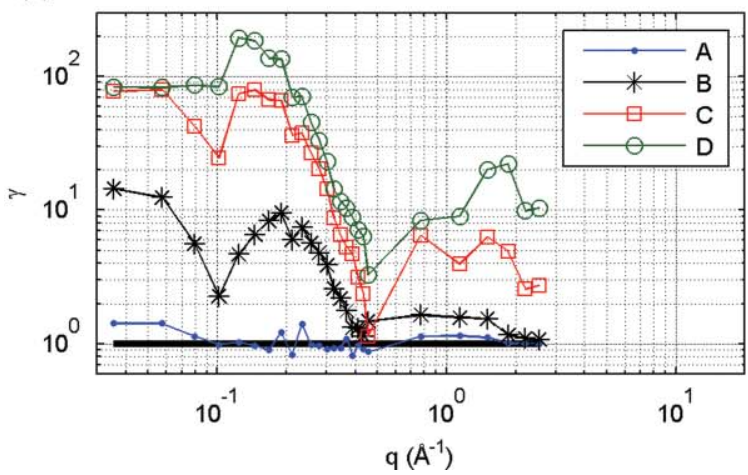

FIG. 9. (Color online) Flame experiment at $5 \mathrm{keV}$ with a time resolution of $1 \mathrm{~s}$. (a) Time trace with the signal from every channel marked to the right. Four different times are also marked: $230 \mathrm{~s}, 490 \mathrm{~s}, 980 \mathrm{~s}$, and $2370 \mathrm{~s} \mathrm{(A,} \mathrm{B,}$ $\mathrm{C}$, and D). (b) The black thick line shows the theoretical scattering for air, and the curves A-D show the scattering intensities as a function of the q-vector for the four different times marked in the time trace. (c) The curves A-D are presented as the ratio of the intensities with respect to the initial scattering condition. The black thick line shows the initial flame condition $(\gamma=0)$.

between $1 \AA^{-1}$ and $2 \AA^{-1}$, which developed to a graphitic structure (close to $1.75 \AA^{-1}$ ) after about $2400 \mathrm{~s}$.

Experiments on burning magnesium powder were performed at beamline D611, MAX-lab. A match was mounted vertically on a holder with the head up and magnesium powder was placed at the bottom of the match. The x-ray beam was passing through the match, just below the head. A spark plug was controlled from the outside of the x-ray hutch to ignite the match. Figure 10 shows a schematic picture of different stages of the experiment: (a) the spark plug was dis-

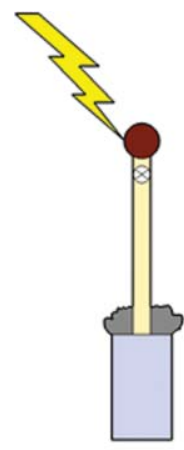

(a)

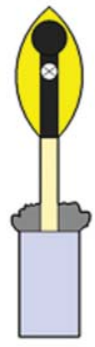

(b)

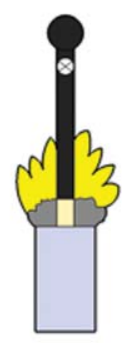

(c)

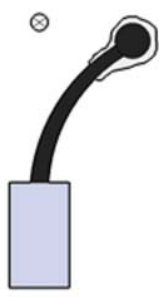

(d)
FIG. 10. (Color online) A match was attached on a holder and the magnesium powder was placed at the bottom of the match. The x-ray beam penetrated the match just below the head. (a) A discharge from a spark plug was produced. (b) The match burned, leaving the top partially carbonized. (c) The magnesium powder started to burn and almost the whole match was carbonized on the outside. (d) Magnesium oxide had formed around the top of the match. The whole match was carbonized and bent.

charged; (b) the upper part of the match was burning and the wood in the probe region was only partially carbonized; (c) the flame front had reached the lowest part of the match and ignited the magnesium powder; (d) all the magnesium had reacted and the match was completely carbonized, bent, and the flame had extinguished.

Figure 11(a) shows the time trace of the ignition, burning of the match and magnesium with a time resolution of $0.1 \mathrm{~s}$. The scattering intensity for the four different times A, B, C, and $\mathrm{D}$ is plotted in Fig. 11(b). The black line without marks is the peak from magnesium oxide, received from the MSCWAXS detector while scanning the wavelength. The angular offsets were $-0.1^{\circ}$ and $0^{\circ}$ for the MSC-SAXS and WAXS detectors, respectively. The spark plug was discharged and the ignition of the match took place between $\mathrm{A}$ and $\mathrm{B}$ and the wood started to burn. The flame front moved down leaving the upper part of the match partially carbonized and the probed region becomes carbonized at B (Fig. 10(b)). Between $\mathrm{B}$ and $\mathrm{C}$ there are changes in the SAXS region, $0.028 \AA^{-1}$ $<\mathrm{q}<0.35 \AA^{-1}$ increases and $0.38 \AA^{-1}<\mathrm{q}<0.70 \AA^{-1}$ decreases, indication chemical and physical changes inside the probe region. At the end of this time period scattering from magnesium oxide starts to appear as the flame front ignites the magnesium powder and an aerosol is formed and reached the probe volume. The magnesium oxide is formed around the upper part of the match and the peak in the scattering curve (q $=2.99 \AA^{-1}$ ) is reached at time $\mathrm{D}$. As the wood burns down to the end position where it is fixed to the holder, the burnt match becomes bent and moves out from the probe volume (Fig. 10(d)).

The MSC-SAXS detector has also been used to monitor drops in a levitator while they were evaporating. Measurements on different drops in operation mode I were performed at beamline I911-5, MAX-lab. The photon energy was $13.6 \mathrm{keV}$ and sample averaging times of $1 \mathrm{~s}, 0.1 \mathrm{~s}$, and $0.02 \mathrm{~s}$ were used. Figure 12 shows a time trace of the SAXS from the final stages of an evaporation toluene drop suspended in an ultrasonic levitator. The time resolution was $0.1 \mathrm{~s}$. The main evaporation observed from the SAXS 


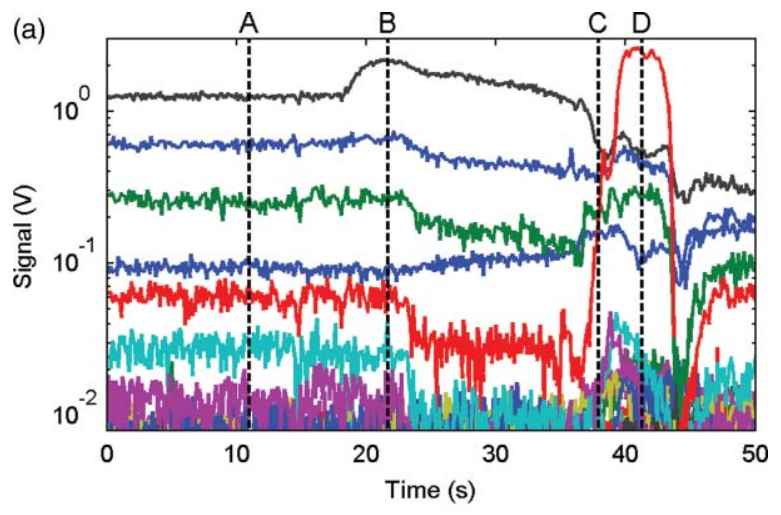

(b)

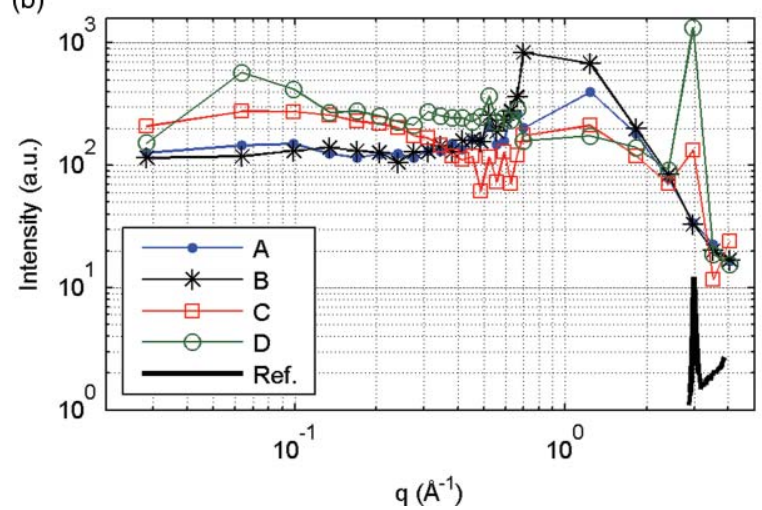

FIG. 11. (Color online) Experimental data from burning magnesium at $8 \mathrm{keV}(\lambda=1.55 \AA)$ with a time resolution of $0.1 \mathrm{~s}$. (a) Time trace with four different times marked: 10.9 s, 21.6 s, 37.9 s, and 41.2 s (A, B, C, and D). (b) The scattered intensity as a function of the q-vector for the four different times marked in the time trace. The black reference line is the peak from magnesium oxide.

occurs between $8 \mathrm{~s}$ (A) and $25 \mathrm{~s}$ (B), when the whole drop had disappeared. The signal level for the toluene drop before evaporation is marked (I) as well as the signal level for background air (II). Interesting to note are also the correlated fluctuations in the scattering intensities from the different

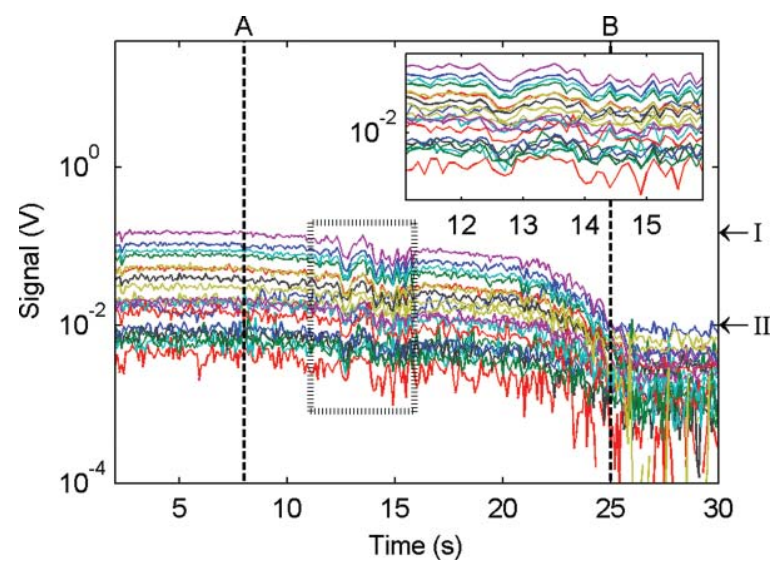

FIG. 12. (Color online) The time trace of a toluene drop in a levitator. Maximum sampling rate was used and software averaging every $0.1 \mathrm{~s}$. The final stages of evaporation occurred between $8 \mathrm{~s}$ (A) and $25 \mathrm{~s}$ (B). The level I shows the signal level for the toluene drop before evaporation and the level II shows the signal level for background air. The inset in the figure shows the time trace between $11 \mathrm{~s}$ and $16 \mathrm{~s}$. channels. These are not due to detector noise but to droplet dynamics during evaporation. Although these features cannot yet be explained they are an interesting subject to study in coming experiments, in particular in relation to the behavior of evaporation characteristics interesting for fuel sprays under isothermal and burning conditions in combustion.

\section{DISCUSSION}

The MSC-SAXS and WAXS detector allow conducting time-resolved measurements over a time span from seconds to hours with a dynamic range of 24-bits. The sampling system performs continuous measurements without readout dead time, thanks to the dual core processor that enables sampling and processing of the data in real time. The acquisition time might be limited depending on the number of used channels and by the number of cores in the computer. If the processing time (the time it takes to process the data by one of the two methods) is longer than the sampling interval, the computer starts to buffer data, and the acquisition time is limited by available physical memory. Sampling intervals of $0.1 \mathrm{~s}$ have been used without problems but experiments with a sampling time of $20 \mathrm{~ms}$ could only be run for a few minutes. However, by directly storing the raw data and processing it afterwards experiments on fast single events become possible. The time resolution is then determined by the decay time of the scintillator and the pre-amplifier, which is in total $35 \mu \mathrm{s}$. If faster scintillators are used in combination with a shorter time constant for the pre-amplifier, the sampling rate of the data acquisition cards, which is close to $5 \mu \mathrm{s}$, will set the limit for the time resolution. Experiments with time resolutions higher than $20 \mathrm{~ms}$ were not performed due to the low signal-to-noise ratio, caused by the limited photon fluxes provided by the $\mathrm{x}$-ray beam. However, experiments with more intense $\mathrm{x}$-ray beams will enable an increased time resolution.

The detectors can be used for slow dynamic processes up to hours, e.g., the soot formation in a flame and also for faster dynamic processes on sub-millisecond time scale. Due to the continuous sampling, changes in the scattering pattern can be detected directly and no events in the dynamic process will be lost through readout time. For steady state processes the q-range can be extended by scanning the wavelength or rotating the detectors. The q-resolution is then limited by the accuracy of the wavelength scan and the rotator device.

Every channel selects a relatively narrow spatial angle, which makes background scattering relatively easy to suppress. However, every channel observes different scattering volumes and the largest variation is within the MSC-SAXS detector. This can become a problem when performing experiments on extended objects of non-homogeneous distributions since the smallest channels on the MSC-SAXS detector detects scattered photons from a larger volume than the other channels. The depth of view has both been calculated and measured for the MSC-SAXS detector (cf. Fig. 7) and the experimental result agrees well with the calculations, except for the first angle. This channel is very sensitive to the angular offset (cf. Fig. 2). This issue will be further investigated in the future. 
The chopper and the OLBS software are used to reduce noise from the signal during the experiments. In our case the noise is mainly visible light leaking into the MSC-WAXS detector, which is a prototype version for a newer detector with more channels, on the way of being built. Modulation, alternatively with another method, can be used to reduce electric noise. Scattering noise from outside the measurements volume can also be reduced if modulation is applied just to the measured region. The advantage of the OLBS algorithm is the detection of the signal amplitude regardless of the offset, while the AV method detects wrong signal amplitude if an offset due to noise is present. The OLBS algorithm is much more time consuming than the $\mathrm{AV}$ but if a time resolution of $0.1 \mathrm{~s}$ is enough for the experiments, this algorithm can be used without buffering problem.

The MSC-SAXS detector covers a range of $0.022 \AA^{-1}$ $<\mathrm{q}<0.44 \AA^{-1}$ and the MSC-WAXS detector covers $0.77 \AA^{-1}<\mathrm{q}<2.53 \AA^{-1}$ for $5 \mathrm{keV}$. For higher energies the range will be shifted towards higher q-values. The gap between the MSC-SAXS and WAXS detectors will be covered by a new detector with 20 channels.

The detectors have successfully been used for flame studies, burning of magnesium powder and for the dynamics of evaporating drops. The results from the drop studies demonstrate that the detectors can be used for spray studies and to study the evaporation and structural changes of drops in the future.

The detectors are today part of a mobile set-up which needs about half a meter of space after the x-ray beam exit. The advantage with this compact set-up is the possibility to bring it to different beamlines and in that way get access to various beam properties, depending on the type of experiment that is going to be performed. The MSC-SAXS detector has so far been used at two different beamlines.

\section{CONCLUSIONS}

The performances of a new detector for combined SAXS and WAXS measurements have been demonstrated. It has been used for wavelengths in the range from $5 \mathrm{keV}$ to $13.6 \mathrm{keV}$. There is no dead time for readout and the continuous measurements, with a time resolution of $\sim 100 \mathrm{~ms}$, can extend over hours. The sampling system can also be used for faster events with an acquisition time of minutes and the time resolution is then $35 \mu \mathrm{s}$. In addition, the detector has a high dynamic range and resolution enabling the detection of small changes in the scattering intensity. Different applications have been demonstrated, e.g., measurements on nonrepetitive events.

\section{ACKNOWLEDGMENTS}

We acknowledge Lennart Österman for building the preamplifier, Pelle Steen for LabVIEW support, Soenke Seifert for samples and reference measurements of silver behenate, Maxim Tchaplyguine for magnesium samples, and Rutger Lorensson for the ignition system used for the magnesium oxide experiments. The support from Crafoordska Stiftelsen and Carl Tryggers Stiftelsen are acknowledged for equipment support. The Swedish Energy Agency, the Swedish Research Council, the Linneus Grant by the Swedish Research Council and the Centre for Combustion Science and Technology are also greatly acknowledged.

${ }^{1}$ S. Mazumder, R. Dey, M. K. Mitra, S. Mukherjee, and G. C. Das, J. Nanomater. 2009, 815734 (2009).

${ }^{2}$ A. Maliakal, H. Katz, P. M. Cotts, S. Subramoney, and P. Mirau, J. Am. Chem. Soc. 127, 14655 (2005).

${ }^{3}$ F. E. Kruis, H. Fissan, and A. Peled, J. Aerosol Sci. 29, 511 (1997).

${ }^{4}$ N. H. Chou, X. Ke, P. Schiffer, and R. E. Schaak, J. Am. Chem. Soc. 130, 8140 (2008).

${ }^{5}$ L. Yu, A. Banarjee, and H. Matsui, J. Am. Chem. Soc. 125, 14837 (2003).

${ }^{6}$ M. G. Lines, J. Alloys Compd. 449, 242 (2008).

${ }^{7}$ G. G. Wallance, J. Chen, A. J. Mozer, M. Forsyth, D. R. MacFarlane, and C. Wang, Mater. Today 12(6), 20 (2009).

${ }^{8}$ R. Mueller, H. K. Kammler, S. E. Pratsinis, A. Vital, G. Beaucage, and P. Burtscher, Powder Technol. 140, 40 (2004).

${ }^{9}$ H. K. Kammler, R. Jossen, P. W. Morrison, Jr., S. E. Pratsinis, and G. Beaucage, Powder Technol. 135-136, 310 (2003).

${ }^{10}$ B. M. Kumfer, K. Shinoda, B. Jeyadevan, and I. M. Kennedy, J. Aerosol Sci. 41, 257 (2010).

${ }^{11}$ M. Katzer, A. P. Weber, and G. Kasper, Aerosol Sci. 32, 1045 (2001).

${ }^{12}$ J. Polte, R. Erler, A. F. Thünemann, S. Sokolov, T. T. Ahner, K. Rademann, F. Emmerling, and R. Kraehnert, ACS Nano 4, 1076 (2010).

${ }^{13}$ J. B. A. Mitchell, J. L. LeGarrec, M. Sztucki, T. Narayanan, V. Dikhtyar, and E. Jerby, Phys. Rev. Lett. 100, 065001 (2008).

${ }^{14}$ D. J. Tobler, S. Shaw, and L. G. Benning, Geochim. Cosmochim. Acta 73, 5377 (2009)

${ }^{15}$ W. A. England, Combust. Sci. Technol. 46, 83 (1986).

${ }^{16}$ J. P. Hessler, S. Seifert, and R. E. Winans, Proc. Combust. Inst. 29, 2743 (2002).

${ }^{17}$ J. P. Hessler, S. Seifert, R. E. Winans, and T. H. Fletcher, Faraday Discuss. 119, 395 (2001).

${ }^{18}$ S. di Stasio, J. B. A. Mitchell, J. L. LeGarrec, L. Biennier, and M. Wulff, Carbon 44, 1267 (2006).

${ }^{19}$ J. B. A. Mitchell, J. Courbe, A. I. Florescu-Mitchell, S. di Stasio, and T. Weiss, J. Appl. Phys. 100, 124918 (2006).

${ }^{20}$ M. Sztucki, T. Narayanan, and G. Beaucage, J. Appl. Phys. 101, 114304 (2007).

${ }^{21}$ G. Beaucage, H. K. Kammler, R. Mueller, R. Strobel, N. Agashe, S. E. Pratsinis, and T. Narayanan, Nature Mater. 3, 370 (2004).

${ }^{22}$ F. Ossler and J. Larsson, Chem. Phys. Lett. 387, 367 (2004).

${ }^{23}$ F. Ossler and J. Larsson, J. Appl. Phys. 98, 114317 (2005).

${ }^{24}$ J. Bolze, D. Pontoni, M. Ballauff, T. Narayanan, and H. Cölfen, J. Colloid Interface Sci. 277, 84 (2004).

${ }^{25}$ Y. Shinohara, N. Kawasaki, S. Ueno, I. Kobayashi, M. Nakajima, and Y. Amemiya, Phys. Rev. Lett. 94, 097801 (2005).

${ }^{26}$ A. Mahendrasingam, D. J. Blundell, A. K. Wright, V. Urban, T. Narayanan, and W. Fuller, Polymer 45, 5641 (2004).

${ }^{27}$ V. Urban, P. Panine, C. Ponchut, P. Boesecke, and T. Narayanan, J. Appl. Cryst. 36, 809 (2003).

${ }^{28}$ P. Kraft, A. Bergamaschi, Ch. Brönnimann, R. Dinapoli, E. F. Eikenberry, H. Graafsma, B. Heinrich, I. Johnson, M. Kobas, A. Mozzanica, C. M. Schlepütz, and B. Schmitt, IEEE Trans. Nucl. Sci. 56, 758 (2009).

${ }^{29}$ A. Bergamaschi, C. Broennimann, E. Eikenberry, B. Henrich, M. Kobas, P. Kraft, and B. Schmitt, Proceedings of the 16th International Workshop on Vertex detectors, Lake Placid, 2007.

${ }^{30}$ A. Bergamaschi, A. Cervellino, R. Dinapoli, F. Gozzo, B. Henrich, I. Johnson, P. Kraft, A. Mozzanica, B. Schmitt, and X. Shi, Nucl. Instrum. Methods Phys. Res. A 604, 136 (2009).

${ }^{31}$ P. M. De Lurgio, J. P. Hessler, J. T. Weizeorick, A. S. Kreps, M. J. Molitsky, I. Naday, G. R. Drake, and G. Jennings, IEEE Nucl. Sci. Symp. Conf. Rec. 2, 1215 (2005).

${ }^{32}$ P. M. De Lurgio, G. R. Drake, A. S. Kreps, G. Jennings, J. T. Weizeorick, M. J. Molitsky, I. Naday, and J. P. Hessler, IEEE Trans. Nucl. Sci. 57, 1706 (2010).

${ }^{33}$ F. Ossler, S. E. Canton, and J. Larsson, Carbon 47, 3498 (2009).

${ }^{34}$ L. Jonsson, Master's thesis, Lund University, Lund, 2009.

${ }^{35}$ M. A. Aramendía, J. A. Benítez, V. Borau, C. Jiménez, J. M. Marinas, J. R. Ruiz, and F. Urbano, Langmuir 15, 1192 (1999). 